\section{Motor imagery and its effect on complex regional pain syndrome: an integrative review}

\author{
Nélio Silva de Souza, 1,2 Ana Carolina \\ Gomes Martins, 1,3 Victor Hugo do Vale \\ Bastos,2,4,5 Marco Orsini,2,6 \\ Marco Antônio A. Leite, 7,8 \\ Silmar Teixeira,9-11 Bruna Velasques, 11,12 \\ Pedro Ribeiro, 11,12 Juliana Bittencourt, 11 \\ André Palma da Cunha Matta, 4 \\ Pedro Moreira Filho 4 \\ 1 University Center Serra dos Órgãos, \\ Teresópolis; 2Science of Rehabilitation, \\ Centro Universitário Augusto Motta, Rio \\ de Janeiro; 3 Integrated Health of Women \\ and Children, Fluminense Federal \\ University, Niterói; 4Department of \\ Neurology, Antônio Pedro University \\ Hospital, Fluminense Federal University, \\ Niterói; ${ }^{5}$ Biomedical Sciences stricto \\ sensu, Federal University of Piauí; \\ 6Severino Sombra University Center, \\ Medicine Department, Vassouras, Rio de \\ Janeiro; 7Fluminense Federal University \\ Medical School, Niterói; 8Movement \\ Disorders Unit, Antônio Pedro University \\ Hospital, Fluminense Federal University, \\ Niterói, 9 Brain Mapping and Plasticity \\ Laboratory, ${ }^{10}$ Brain Mapping and \\ Functionality Laboratory, Federal \\ University of Piauí, Parnaíba; ${ }^{11 B r a i n}$ \\ Mapping and Sensory Motor Integration, \\ Institute of Psychiatry of Federal \\ University of Rio de Janeiro; 12Institute \\ of Applied Neuroscience, Rio de Janeiro, \\ Brazil
}

\section{Abstract}

The motor imagery (MI) has been proposed as a treatment in the complex regional pain syndrome type 1 (CRPS-1), since it seems to promote a brain reorganization effect on sensory-motor areas of pain perception. The aim of this paper is to investigate, through an integrative critical review, the influence of MI on the CRPS-1, correlating their evidence to clinical practice. Research in PEDro, Medline, Bireme and Google Scholar databases was conducted. Nine randomized controlled trials (level 2), 1 non-controlled clinical study (level 3), 1 case study (level 4), 1 systematic review (level 1), 2 review articles and 1 comment (level 5) were found. We can conclude that MI has shown effect in reducing pain and functionality that remains after 6 months of treatment. However, the difference between the MI strategies for CRPS-1 is unknown as well as the intensity of mental stress influences the painful response or effect of MI or other peripheral neuropathies.

\section{Introduction}

The motor imagery (MI) or mental simulation of movements can be defined as the act of mentally play an action without executing it.1,2. Some properties observed during the execution of movements are also present during MI,1,3-7 because there are similarities in the mental states during execution and imagination of the same movement. 1,8 With advances in neuroimaging techniques, it was possible to demonstrate the existence of overlap between the neural circuits accessed during MI and execution of the same movement.9-11 The circuits involved both in implementation and in the simulation of a task include: the supplementary motor area; The primary motor cortex (M1); The parietal cortex; basal ganglia and cerebellum. ${ }^{9,11-14}$ Some of these brain areas have shown changes in patients with complex regional pain syndrome (CRPS) during execution and MI of the same movement.15,16

The CRPS affects $1-5 \%$ of individuals with a history of trauma in the member, ${ }^{17-21}$ leading to chronic changes in different sensorimotor levels and the consequent disruption of exteroceptive acuity (tactile information).22,23 The CRPS type 1 is diagnosed when there is no neural damage while CRPS type 2 is associated with nerve injury. 19,22,24 The etiology of CRPS remains unknown, with heterogeneous symptoms and different levels in the sensory (pain and hyperalgesia), autonomic (changes in temperature, color and sweating in the skin) and motor (weakness or loss of range of motion). ${ }^{20,22,24}$ Although their behavior is varied, the CRPS has three patterns: i) increased inflammatory response; ii) vasomotor dysfunction, and iii) adaptive changes in neural plasticity. 25

The CRPS type 1 (SCR-1) is involved in similar cortical abnormalities observed in phantom pain and after a cerebrovascular stroke (CVA). ${ }^{26}$ This condition causes changes to the peripheral level (high synaptic activity, increased neurogenic inflammatory response and reduction of proprioceptive reflexes) and central (disturbances in the processing of sensory-motor cortex and body base schema), ${ }^{26}$ leading to changes in the proprioceptive representation (sensory and motor cortex) the following involved, promoting inconsistencies between motor control and sensory feedback and subsequent sensorimotor disorganization. $15,27,28$ Recently, the application of Program Motor Imagery (PIM) has been proposed in patients with CRPS type 1,26,29-32
Correspondence: Marco Orsini, Department of Neurosurgery, Universidade Federal Fluminense, 95 Tavares de Macedo street, 902 Niterói, RJ, ZIP 24220-211 Brazil.

E-mail: orsinimarco@hotmail.com

Key words: Motor imagery; complex regional pain syndrome, peripheral neuropathic pain.

Contributions: the authors contributed equally.

Conflict of interest: the authors declare no potential conflict of interest.

Received for publication: 21 April 2015.

Revision received: 21 August 2015.

Accepted for publication: 12 November 2015.

This work is licensed under a Creative Commons Attribution NonCommercial 3.0 License (CC BYNC 3.0).

(C) Copyright N.S. de Souza et al., 2015

Licensee PAGEPress, Italy

Neurology International 2015; 7:5962

doi:10.4081/ni.2015.5962

showing changes in the processing of brain activity in both areas, sensory perception of pain (S1 and S2) ${ }^{15,16}$ and in motor areas (M1), ${ }^{15,33}$ because the pain can lead the different stages of sensorimotor disorganization. ${ }^{34}$ In this context, the purpose of this study was to investigate, by an integrative critical review, the influence of MI on the CRPS-1, correlating their evidence with the application in clinical practice.

\section{Materials and Methods}

This study is characterized by an integrative literature review that aims to gather, critically evaluate according to the level of evidence of the 0xford Centre for Evidence-Based Medicine (http://www.cebm.net/index.aspx?0=5653) and a synthesis result to be related to the clinical practice. For searching the data source we used the following combined keywords or not: motor imagery; complex regional pain syndrome and peripheral neuropathic pain.

\section{Data sources, inclusion and exclusion criteria}

The results of the search from each database will be detailed in Table 1 .

\section{PubMed/Medline}

Among the 25 articles 10 were selected. From these, 15 studies were excluded because of their title involving M or CRPS only, but not 
the two associated in the same study. Moreover, these studies were excluded because these were about reviews and/or general treatment of CRPS, which included MI as a non-invasive or non-pharmacological treatment, citing the main works described in Supplementary Tables S1-3 of this study.

\section{Google Scholar}

From the 124 articles found, 5 were selected. We excluded 117 items by their title involving MI or CRPS only, but not the two associated in the same study. In addition, we found several books on one of the subjects and, therefore they were excluded from the selection.

\section{Searching results}

Different types of study were found in this integrative review. In Supplementary Table S1 there are 9 papers performing randomized controlled clinical trials (evidence level 2). Supplementary Table S2 presents 1 uncontrolled clinical study (Level of evidence 3 ) and 1 case study (evidence level 4). Finally, in Supplementary Table S3 there is the first systematic review study (evidence level 1), 2 review articles and 1 comment (evidence level 5) on the proposed topic.

\section{Motor imagery}

The MI may be performed using two different strategies: i) visual and ii) kinesthetic. ${ }^{2}$ In the first one, the participant mentally visualizes a movement being performed by him or by another person using the visual perception of the imagined movement. In the second strategy, the participant uses the kinesthetic perception of the imagined movement from somaticmotor information, involving part of the mechanisms used in the preparation and programming of the action.2,6,14 Neuroimaging studies have shown that while there is a common neural substrate between these two strategies (visual and kinesthetic),1,8 distinct neural circuits are activated in each of them to simulate the same task. ${ }^{2,9}$ So far, the work done with CRPS using the MI has not taken into account these two types of strategies (Supplementary Tables S1-3) and it may exist different effects between them. For example, it has been shown that the MI kinesthetic has greater sway center of pressure $(\mathrm{CoP})$ compared with the visual MI in different tasks ${ }^{35-37}$ and this effect has been correlated with the intensity of the imagined movement, ${ }^{38}$ which is another point that has not been explored in studies with CRPS-1. Therefore, it can be expected that MI strategies (visual and kinesthetic) may also have different effects on pain perception in the CRPS-1 reorganization.

\section{Complex regional pain syndrome and sensorimotor adaptation to painful stimuli}

In general, the pain stimulus (fiber type III and IV) may lead to an increase in the excitability of motoneurons, accompanied by a decrease in cortical excitability. 39 This pain is a noxious stimulus that can lead to disruption in sensorimotor planning in different clinical conditions, such as low back pain associated with changes in postural control;40 musculoskeletal disorders ${ }^{41,42}$ and peripheral neuropathic pain, specifically in CRPS. 15 People in pain have slower response time to determine the hand laterality compared to individuals without pain, ${ }^{41}$ indicating a disruption in sensorimotor planning.

Currently, it has been proposed a new theory of adaptation to pain, 34 contrasting the two most traditional theories, which are the theory of pain-spasm-pain cycle and the theory of adaptation to pain. 43,44 This recent theory proposes that 5 stages of sensorimotor adaptation to pain stimuli occur: i) redistribution of intraand intermuscular activity; ii) changes in the mechanical behavior of the individual (change in motor recruitment order); iii) the pain stimulus or injury projects a threatening effect in relation to aversive stimulus; iv) sensorimotor changes at multiple encephalic levels and v) sensorimotor consequences due to functional adaptations in short and long term. ${ }^{34}$

In this context, low back pain seems to promote disruption in human motor cortex, ${ }^{40}$ leading to physiological changes in motor recruitment order. 45 The lumbar spine stabilization exercises are able to redeem this physiological muscle recruitment order and decrease pain in the region because the execution of a motor command can facilitate the inhibition of the chronically sensitized spinal nociceptive projections. ${ }^{40,46}$ Similarly, MI surrounds part of the circuit used in the preparation and programming of a motor act ${ }^{9-11}$ and its use in patients with CRPS has shown a possi- ble brain reorganization effect, ${ }^{26,29-32}$ as will be detailed below.

\section{Use of motor imagery to brain reorganization in complex regional pain syndrome type 1}

For the implementation or mental simulation of the same movement, proactive intelligence sensory system is required, 14 including pain information, ${ }^{41}$ which can lead to a disruption in areas related to the sensory-motor planning, ${ }^{15}$ leading to changes in brain activity, specifically in pain processing areas (S1 and S2). ${ }^{15,16}$ It seems that the mental training using the PMI can promote a reorganization of these areas, 15,16 leading to reduction of pain and edema in patients with peripheral neuropathy, specifically with CRPS-1.26,29-32

The MI technique has had a significant effect of reducing pain in CRPS-1 after hand fracture, $26,29-32$ decreasing the intensity of pain and promoting increased functionality, ${ }^{21,26,31,32,47,48}$ that can be maintained even after 6 months of intervention (see Table 1). ${ }^{32}$ However, some individuals, who have suffered spinal cord injury, ${ }^{49}$ or chronic CRPS in the arm, ${ }^{29}$ cannot benefit from the use of MI because there are still flaws in their use..$^{50}$ It is possible that the participants intensity variations of the mental effort during the MI can promote these divergent results. For example, Lemos et al. ${ }^{35}$ showed that only high levels of intensity of the imagined movement (of strategic kinesthetic MI) are able to promote greater variability of the center of gravity oscillation (CoG), compared to participants with low intensity levels of the imagined movement. ${ }^{38}$

The effect of MI strategies (visual and kinesthetic) ${ }^{2}$ in CRPS-1 was further compared in the same group. To date, only visual MI have been used (visualizing figures) 26,29-32 $^{\text {or kinesthetic }}{ }^{32}$ in isolation (Table 1). It seems that the MI kinesthetic strategy can specifically modulate pain perception, similar to the effects observed during exercise, because there are similarities between the execution and imagination in the same exercise. ${ }^{1,6}$ It is possible that the MI kinesthetic promote changes in three levels of pain perception: i) immediately, with the release of -enkephalin and met-enkephalin similar to physical exercise; 51 ii) modulating the perception of pain in the dorsal horn of the spinal cord and cerebral cortex, inhibiting the

Table 1. Search results in databases.

Database Articles found/selected

PubMed/Medline 25/10
Authors (year)

Moseley (2004); Moseley (2005); Moseley (2006);

Moseley et al. (2008); Gieteling et al., (2008); Maihöfner \& Speck (2012);

Johnson et al. (2012); Lagueux et al. (2012); Walz et al. (2013)

Scholar Google 124/4 Gustin et al. (2008); Coslett et al. (2010); Bowering et al. (2013); Katholi et al. (2014) 
pain pathway (type A fiber and/or C and spinal pathway) ${ }^{52,53}$ and iii) the long term, for at least 6 months of training 32 , the reorganization of the sensorimotor cortex may occur, 33 through the unmasking processes and synaptic strengthening and/or neural plasticity.

\section{Conclusions}

The articles on the subject have shown satisfactory levels of evidence (levels 1 and 2 mostly) with publications in journals of good international visibility (Table 1). It seems that the effect of CRPS in the MI is connected to encephalic reorganization promoted by mental simulation of the movement. However, no work until the present moment compared the effect of MI modalities (visual and kinesthetic) or even the intensity of mental effort in this clinical condition. Furthermore, there is no MI work involving other conditions of peripheral neuropathic pain (post polio syndrome, diabetes mellitus, etc.), although it is possible that the MI may also have a similar effect under these conditions.

\section{References}

1. Jeannerod M. The representing brain: neural correlates of motor intention and imagery. Cambridge Univ Press 1994;17: 187-245.

2. Sirigu A, Duhamel JR. Motor and visual imagery as two complementary but neurally dissociable mental processes. J Cogn Neurosci 2001;13:910-9.

3. Decety J, Jeannerod M, Prablanc C. The timing of mentally represented actions. Behav Brain Res 1989;34:35-42.

4. Parsons LM. Temporal and kinematic properties of motor behavior reflected in mentally simulated action. J Exp Psychol Hum Percept Perform 1994;20:709-30.

5. Jeannerod M, Decety J. Mental motor imagery: a window into the representational stages of action. Curr Opin Neurobiol 1995; 5:727-32.

6. Decety J. The neurophysiological basis of motor imagery. Behav Brain Res 1996;77: 45-52.

7. Jeannerod M. Mental imagery in the motor context. Neuropsychologia 1995;33:1419-32.

8. Decety J. Do imagined and executed actions share the same neural substrate? Brain Res Cogn Brain Res 1996;3:87-93.

9. Ruby P, Decety J. Effect of subjective perspective taking during simulation of action: a PET investigation of agency. Nat Neurosci 2001;4:546-50.

10. Guillot A, Collet C, Nguyen VA, et al. Brain activity during visual versus kinesthetic imagery: an fMRI study. Hum Brain Mapp 2009;30:2157-72.

11. Guillot A, Collet C, Nguyen VA, et al. Functional neuroanatomical networks associated with expertise in motor imagery. Neuroimage 2008;41:1471-83.

12. Lotze M, Montoya P, Erb M, et al. Activation of cortical and cerebellar motor areas during executed and imagined hand movements: an fMRI study. J Cogn Neurosci 1999;11:491-501.

13. Gerardin E, Sirigu A, Lehéricy S, et al. Partially overlapping neural networks for real and imagined hand movements. Cereb Cortex 2000;10:1093-104.

14. Jeannerod M. Neural simulation of action: a unifying mechanism for motor cognition. Neuroimage 2001;14:S103-9.

15. Gieteling EW, van Rijn M, de Jong BM, et al. Cerebral activation during motor imagery in complex regional pain syndrome type 1 with dystonia. Pain 2008; 134:302-9.

16. Walz AD, Usichenko T, Moseley GL, Lotze M. Graded motor imagery and the impact on pain processing in a case of CRPS. Clin J Pain 2013;29:1.

17. Birklein F, Handwerker HO. Complex regional pain syndrome: how to resolve the complexity? Pain 2001;94:1-6.

18. Woolf CJ, Mannion RJ. Neuropathic pain: aetiology, symptoms, mechanisms, and management. Lancet 1999;353:1959-64.

19. Shah A, Kirchner JS. Complex regional pain syndrome. Foot Ankle Clin 2011;16:3 51-66.

20. Katholi BR, Daghstani SS, Banez G, Brady KK. Noninvasive Treatments for pediatric complex regional pain syndrome: a focused review. Am Acad Phys Med Rehabil 2014;6:926-33.

21. Lagueux E, Charest J, Lefrançois-Caron E, et al. Modified graded motor imagery for complex regional pain syndrome type 1 of the upper extremity in the acute phase. Int J Rehabil Res 2012;35:138-45.

22. Maihöfner C, Seifert F, Markovic K. Complex regional pain syndromes: new pathophysiological concepts and therapies. Eur J Neurol 2010;17:649-60.

23. Pleger B, Ragert P, Schwenkreis P, et al. Patterns of cortical reorganization parallel impaired tactile discrimination and pain intensity in complex regional pain syndrome. Neuroimage 2006;32:503-10.

24. Maihöfner C, Speck V. Graded motor imagery for complex regional pain syndrome: where are we now? Eur J Pain 2012; 16:461-2.

25. Marinus J, Moseley GL, Birklein F, et al. Clinical features and pathophysiology of complex regional pain syndrome. Lancet Neurol 2011;10:637-48.
26. Moseley GL. Graded motor imagery is effective for long-standing complex regional pain syndrome: a randomised controlled trial. Pain 2004;108:192-8.

27. Harris AJ. Cortical origin of pathological pain. Lancet 1999;354:1464-6.

28. Juottonen K, Gockel M, Silén T, et al. Altered central sensorimotor processing in patients with complex regional pain syndrome. Pain 2002;98:315-23.

29. Moseley GL, Zalucki N, Birklein F, et al. Thinking about movement hurts: the effect of motor imagery on pain and swelling in people with chronic arm pain. Arthritis Care Res 2008;59:623-31.

30. Moseley GL. Imagined movements cause pain and swelling in a patient with complex regional pain syndrome. Neurology 2004;62:1644.

31. Moseley GL. Is successful rehabilitation of complex regional pain syndrome due to sustained attention to the affected limb? A randomised clinical trial. Pain 2005;114: 54-61.

32. Moseley GL. Graded motor imagery for pathologic pain: a randomized controlled trial. Neurology 2006;67:2129-34.

33. Flor $\mathrm{H}$. The functional organization of the brain in chronic pain. Prog Brain Res 2000;129:313-22.

34. Hodges PW, Tucker K. Moving differently in pain: a new theory to explain the adaptation to pain. Pain 2011;152:S90-8.

35. Rodrigues EC, Lemos T, Gouvea B, et al. Kinesthetic motor imagery modulates body sway. Neuroscience 2010;169:743-50.

36. Grangeon M, Guillot A, Collet C. Postural control during visual and kinesthetic motor imagery. Appl Psychophysiol Biofeedback 2011;36:47-56.

37. Rodrigues EC, Imbiriba LA, Leite GR, et al. Mental stimulation strategy affects postural control. Rev Bras Psiquiatr 2003;25:33-5.

38. Lemos T, Souza NS, Horsczaruk CHR, et al. Motor imagery modulation of body sway is task-dependent and relies on imagery ability. Front Hum Neurosci 2014;8:290-9.

39. Martin PG, Weerakkody N, Gandevia SC, Taylor JL. Group III and IV muscle afferents differentially affect the motor cortex and motoneurones in humans. J Physiol 2008;586:1277-89.

40. Tsao H, Galea MP, Hodges PW. Reorganization of the motor cortex is associated with postural control deficits in recurrent low back pain. Brain 2008;131: 2161-71.

41. Coslett HB, Medina J, Kliot D, Burkey AR. Mental motor imagery indexes pain: the hand laterality task. Eur J Pain 2010;14: 1007-13.

42. Pelletier R, Higgins J, Bourbonnais D. Is neuroplasticity in the central nervous system the missing link to our understanding 
of chronic musculoskeletal disorders? BMC Musculoskelet Disord 2015;16:1-13.

43. Roland MO. A critical review of the evidence for a pain-spasm-pain cycle in spinal disorders. Clin Biomech 1986;1:102-9.

44. Lund JP, Donga R, Widmer CG, Stohler CS. The pain-adaptation model: a discussion of the relationship between chronic musculoskeletal pain and motor activity. Can J Physiol Pharmacol 1991;69:683-94.

45. Hodges PW, Richardson CA. Contraction of the abdominal muscles associated with movement of the lower limb. Phys Ther 1997;77:132-42.

46. Porreca F, Ossipov MH, Gebhart GF. Chronic pain and medullary descending facilitation. Trends Neurosci 2002;25:319-25.

47. Coslett HB, Medina J, Kliot D, Burkey AR. NIH public access 2011;14:1007-13.

48. Bowering KJ, Connell NEO, Tabor A, et al. The effects of graded motor imagery and its components on chronic pain: a systematic review and meta-analysis. J Pain 2013;14:3-13.

49. Gustin SM, Wrigley PJ, Gandevia SC, et al. Movement imagery increases pain in people with neuropathic pain following complete thoracic spinal cord injury. Pain 2008;137:237-44.

50. Johnson S, Hall J, Barnet S, et al. Using graded motor imagery for complex regional pain syndrome in clinical practice: failure to improve pain. Eur J Pain 2012;16: $550-61$.

51. Howlett TA, Tomlin S, Ngahfoong L, et al. Release of beta endorphin and metenkephalin during exercise in normal women: response to training. Clin Res Educ 1984;288:1950-2.

52. Woolf CJ. Central sensitization. Anesthesiology 2007;106:864-7.

53. Gilron I, Watson CPN, Cahill CM, Moulin DE. Neuropathic pain: a practical guide for the clinician. CMAJ 2006;175:265-75. 